\title{
RESEARCH ARTICLE \\ Fundamental Theorem of Asset Pricing under fixed and proportional transaction costs
}

\author{
Martin Brown $^{1}$. Tomasz Zastawniak ${ }^{1}$
}

\begin{abstract}
We show that the absence of arbitrage in a model with both fixed and proportional transaction costs is equivalent to the existence of a family of absolutely continuous single-step probability measures, together with an adapted process with values within the bid-ask intervals that satisfies the martingale property with respect to each of the measures. This extends Harrison and Pliska's classical Fundamental Theorem of Asset Pricing to the case of combined fixed and proportional transaction costs.
\end{abstract}

Keywords Arbitrage $\cdot$ Transaction costs $\cdot$ Martingale measure

JEL Classification C00 $\cdot$ C61 $\cdot$ C65 $\cdot$ G10

\section{Introduction}

The Fundamental Theorem of Asset Pricing (FTAP), characterising the absence of arbitrage opportunities as equivalent to the existence of a risk neutral probability measure, has been studied for a large variety of financial market models. The first to establish the result for discrete time models with finite state space were Harrison and Pliska (1981). Dalang et al. (1990) extended the theorem to the case of infinite state space, and Delbaen and Schachermayer $(1994,2006)$ to continuous-time models.

\footnotetext{
$凶$ Tomasz Zastawniak

tomasz.zastawniak@york.ac.uk

Martin Brown

mb885@york.ac.uk

1 Department of Mathematics, University of York, York, UK
} 
The above classical results apply to frictionless models. Harrison and Pliska's result was extended to models with proportional transaction costs by Jouini and Kallal (1995), Kabanov and Stricker (2001) and Ortu (2001). Furthermore, Roux (2011) included interest rate spreads in addition to proportional transaction costs. Similarly, the result by Dalang et al. (1990) involving an infinite state space was extended to models with proportional transaction costs by Zhang and Deng (2002), Kabanov et al. (2002) and Schachermayer (2004). Important milestone achievements concerning the Fundamental Theorem of Asset Pricing in the presence of proportional transaction costs also include, in chronological order, Kabanov (1999), Grigorev (2005), Bouchard (2006), Guasoni (2006), Cherny (2007), Kabanov and Safarian (2009), Guasoni et al. (2010, 2012), Denis and Kabanov (2012), Dolinsky and Soner (2014), Rola (2015), Lépinette and Tran $(2016,2017)$ and Zhao and Lépinette (2018), among others.

Under fixed transaction costs, to our best knowledge, the equivalence between the absence of arbitrage and the existence of risk neutral measures has so far been studied in just one paper, by Jouini et al. (2001). Moreover, characterizations of no-arbitrage conditions for fixed-cost models in terms of separating risk measures (rather than risk neutral measures) are contained as special cases in the work of Lépinette and Tran (2016, 2017).

The long-standing question of extending the Fundamental Theorem of Asset Pricing to cover the situation when both fixed and proportional transaction costs apply simultaneously is addressed in the present paper. We use the term 'combined costs' as shorthand when referring to this case. Such costs are ubiquitous in the markets, hence it is important to be able to characterise the absence of arbitrage in their presence. In Theorem 1 we show that the absence of arbitrage in a market with combined costs is equivalent to the existence of a family of single-step probability measures absolutely continuous with respect to (but not necessarily equivalent to) the physical probability, along with a martingale with respect to such a family of measures (as defined in Sect. 2) and taking values between the bid and ask prices. By doing so, we extend the classical result of Harrison and Pliska (1981) for a finite state space to the case of combined costs. Later on, in Corollary 2 we provide another equivalent condition for the absence of combined-cost arbitrage, namely the existence of an embedded arbitrage-free model with fixed costs.

The technical difficulties inherent in the problem solved here are due to a combination of two factors. On the one hand, under proportional costs, the absence of arbitrage in the full multi-step model is not equivalent to the condition that every single-step submodel should be arbitrage free (even though such an equivalence holds in frictionless models as well as under fixed costs), preventing an argument by reduction to a single step. On the other hand, fixed costs imply that the set of solvent portfolios suffers from the lack of convexity. While these difficulties have been tackled separately in the context of proportional costs and, respectively, fixed costs only, they require fresh ideas to handle their compounded effect. This is achieved in the proof of Theorem 1.

Finally, we refer to the recent work by Lépinette and Tran $(2016,2017)$, in which arbitrage in a non-convex market model with friction (including the case of simultaneous fixed and proportional costs) has been considered, and the absence of asymptotic arbitrage has been characterised by the existence of a so-called equivalent separating 
probability measure. However, no link has been made with risk neutral probabilities, by contrast to the present paper.

\section{Notation and preliminaries}

Let $T$ be a positive integer and let $(\Omega, \Sigma, \mathbb{P})$ be a finite probability space equipped with a filtration $\mathscr{F}=\left(\mathscr{F}_{t}\right)_{t=0}^{T}$. We assume (without loss of generality) that the physical measure $\mathbb{P}$ satisfies the condition $\mathbb{P}(A)>0$ for each non-empty $A \in \mathscr{F}_{T}$, and the sigma-field $\mathscr{F}_{0}$ has a single atom, that is, $\mathscr{F}_{0}=\{\emptyset, \Omega\}$. We refer to the atoms of $\mathscr{F}_{t}$ as the nodes at time $t=0, \ldots, T$, and write $\Lambda_{t}$ for the set of nodes at time $t=0, \ldots, T$. For any non-terminal node $\lambda \in \Lambda_{t}$, where $t=0, \ldots, T-1$, we denote by $\operatorname{succ}(\lambda)$ the set of successor nodes of $\lambda$, that is, nodes $\mu \in \Lambda_{t+1}$ such that $\mu \subset \lambda$.

For each $t=0, \ldots, T$, we can identify any $\mathscr{F}_{t}$-measurable random variable $X$ with a function on $\Lambda_{t}$, and will write $X^{\lambda}$ for the value of $X$ at a node $\lambda \in \Lambda_{t}$.

We shall say that

$$
Q:=\left\{Q_{t}^{\lambda} \mid t=0, \ldots, T-1, \lambda \in \Lambda_{t}\right\}
$$

is a family of absolutely continuous single-step probability measures whenever $Q_{t}^{\lambda}$ is a probability measure defined on the sigma-field

$$
\lambda \cap \mathscr{F}_{t+1}:=\left\{\lambda \cap A \mid A \in \mathscr{F}_{t+1}\right\}
$$

for each $t=0, \ldots, T-1$ and $\lambda \in \Lambda_{t}$. Note that absolute continuity of these measures with respect to $\mathbb{P}$ is automatically ensured by the assumption that $\mathbb{P}(A)>0$ for each non-empty $A \in \mathscr{F}_{T}$. Such a family of measures gives rise to a unique probability measure $\mathbb{Q}$ defined on the sigma-field $\mathscr{F}_{T}$ by

$$
\mathbb{Q}(\lambda):=\prod_{t=0}^{T-1} Q_{t}^{\lambda_{t}}\left(\lambda_{t+1}\right)
$$

for any $\lambda \in \Lambda_{T}$, where $\lambda_{t} \in \Lambda_{t}$ for $t=0, \ldots, T$ is the unique sequence of nodes such that $\lambda_{0} \supset \cdots \supset \lambda_{T}=\lambda$. In general, the family $Q$ is a richer object than the corresponding measure $\mathbb{Q}$ in that it carries more information at those nodes $\lambda$ where $\mathbb{Q}(\lambda)=0$.

Furthermore, we shall say that an adapted process $S$ is a martingale with respect to the family of measures $Q$ if

$$
S_{t}^{\lambda}=\sum_{\mu \in \operatorname{succ}(\lambda)} Q_{t}^{\lambda}(\mu) S_{t+1}^{\mu}
$$

for each $t=0, \ldots, T-1$ and $\lambda \in \Lambda_{t}$. This condition implies that, in particular, $S$ is a martingale (in the usual sense) under the probability measure $\mathbb{Q}$ related to the family $Q$ by (1). In the case when $\mathbb{Q}$ is equivalent to the physical probability $\mathbb{P}$, Eq. (2) is the standard martingale property expressed in terms of the conditional probabilities 
$\mathbb{Q}(\mu \mid \lambda)=Q_{t}^{\lambda}(\mu)$. However, when $\mathbb{Q}(\lambda)=0$ for some node $\lambda$ at time $t$ (in which case $\mathbb{Q}$ is absolutely continuous with respect to $\mathbb{P}$, but not equivalent to $\mathbb{P}$ ), the conditional probability $\mathbb{Q}(\mu \mid \lambda)$ is not well defined. Nonetheless, (2) still applies with $Q_{t}^{\lambda}(\mu)$ taking over the role of the conditional probability.

Families of absolutely continuous single-step probability measures and martingales with respect to such families of measures will be used to characterise the absence of arbitrage in a market model with combined (fixed and proportional) transaction costs; see Theorem 1.

\section{Model with fixed and proportional costs}

Let $A, B$ and $C$ be $\mathbb{R}$-valued processes adapted to the filtration $\mathscr{F}$ such that $0<B \leq$ $A<\infty$ and $0<C<\infty$. We refer to this collection of processes together with the filtration as a combined-cost model, in which $A, B$ play the respective roles of ask and bid stock prices, with $C$ representing fixed transaction costs.

The notions of solvency and self-financing can be formalised as follows in the combined-cost model.

Definition 1 (1) We say that a portfolio $(x, y) \in \mathbb{R}^{2}$ of cash and stock is combinedcost solvent at time $t=0, \ldots, T$ and node $\lambda \in \Lambda_{t}$ when liquidating the stock position leaves a non-negative cash amount

$$
x+B_{t}^{\lambda} y^{+}-A_{t}^{\lambda} y^{-}-C_{t}^{\lambda} \geq 0
$$

after the fixed transaction $\operatorname{cost} C_{t}^{\lambda}$ is met, or when both the cash and stock positions are non-negative to begin with, that is,

$$
x, y \geq 0 .
$$

We denote by $\mathscr{G}_{t}^{\lambda}$ the set of such portfolios $(x, y)$.

(2) We define a combined-cost self-financing strategy as an $\mathbb{R}^{2}$-valued $\mathscr{F}$ predictable process $(X, Y)=\left\{\left(X_{t}, Y_{t}\right)\right\}_{t=0}^{T+1}$ such that

$$
\left(X_{t}^{\lambda}-X_{t+1}^{\lambda}, Y_{t}^{\lambda}-Y_{t+1}^{\lambda}\right) \in \mathscr{G}_{t}^{\lambda}
$$

for each $t=0, \ldots, T$ and $\lambda \in \Lambda_{t}$.

Remark 1 We can also consider the combined-cost liquidation value

$$
L_{t}^{\lambda}(x, y):=x+\left(B_{t}^{\lambda} y^{+}-A_{t}^{\lambda} y^{-}-C_{t}^{\lambda}\right) \mathbf{1}_{y \notin\left[0, C_{t}^{\lambda} / B_{t}^{\lambda}\right]}
$$

of a portfolio $(x, y) \in \mathbb{R}^{2}$ at time $t=0, \ldots, T$ and node $\lambda \in \Lambda_{t}$. Observe that $(x, y) \in \mathscr{G}_{t}^{\lambda}$ is equivalent to $L_{t}^{\lambda}(x, y) \geq 0$. Figure 1 shows a typical set $\mathscr{G}_{t}^{\lambda}$ of combined-cost solvent portfolios. 
Fig. 1 Set $\mathscr{G}_{t}^{\lambda}$ of combined-cost solvent portfolios $(x, y)$ with $A_{t}^{\lambda}=1.5, B_{t}^{\lambda}=0.5, C_{t}^{\lambda}=1.0$

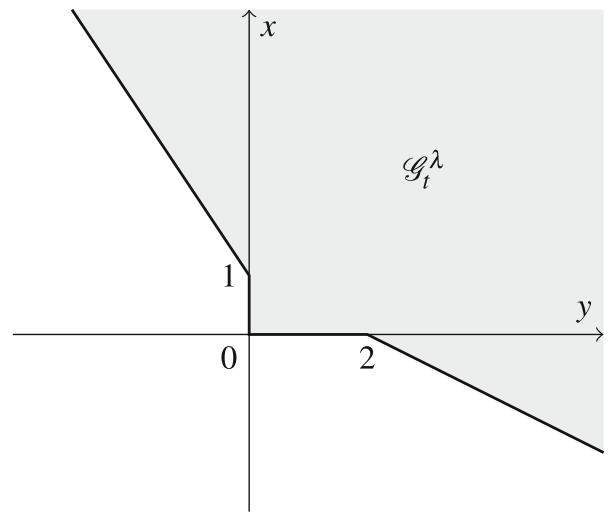

\section{Fundamental Theorem of Asset Pricing under fixed and proportional costs}

Definition 2 A combined-cost self-financing strategy $(X, Y)$ will be referred to as a combined-cost arbitrage opportunity whenever the following conditions hold:

(1) $\left(X_{0}, Y_{0}\right)=(0,0)$,

(2) $X_{T+1} \geq 0$ and $Y_{T+1} \geq 0$,

(3) $X_{T+1}^{\lambda}>0$ for some $\lambda \in \Lambda_{T}$.

Remark 2 The absence of combined-cost arbitrage can be described in terms of the liquidation value introduced in Remark 1. Namely, there is no combined-cost arbitrage opportunity if and only if $L_{T}\left(X_{T}, Y_{T}\right)=0$ for every combined-cost self-financing strategy $(X, Y)$ such that $\left(X_{0}, Y_{0}\right)=(0,0)$ and $L_{T}\left(X_{T}, Y_{T}\right) \geq 0$. This is a direct extension of the classical no-arbitrage (NA) condition studied by Kabanov and Stricker (2001), Schachermayer (2004) and others.

The Fundamental Theorem of Asset Pricing extends to the case of a combined-cost model as follows.

Theorem 1 The following conditions are equivalent:

(1) There is no combined-cost arbitrage opportunity in the model with ask and bid prices $A, B$ and fixed costs $C$;

(2) There exist an adapted process $S$ and a family of absolutely continuous single-step probability measures $Q$ such that $S$ is a martingale with respect to the family $Q$ and $B \leq S \leq A$.

Proof To prove the implication (1) $\Rightarrow(2)$, assume that there is no combined-cost arbitrage opportunity. We begin by constructing two adapted processes $U$ and $V$ by backward induction:

$$
U_{T}^{\lambda}:=A_{T}^{\lambda}, \quad V_{T}^{\lambda}:=B_{T}^{\lambda}
$$


for each $\lambda \in \Lambda_{T}$, and

$$
U_{t-1}^{\lambda}:=\max _{\mu \in \operatorname{succ}(\lambda)}\left(U_{t}^{\mu} \wedge A_{t}^{\mu}\right), \quad V_{t-1}^{\lambda}:=\min _{\mu \in \operatorname{succ}(\lambda)}\left(V_{t}^{\mu} \vee B_{t}^{\mu}\right)
$$

for each $t=1, \ldots, T$ and $\lambda \in \Lambda_{t-1}$.

Having constructed the processes $U$ and $V$, we claim that for each $t=0, \ldots, T-1$ there exist stopping times $\sigma, \tau>t$ such that

$$
U_{t} \geq A_{\sigma}, \quad V_{t} \leq B_{\tau}
$$

We prove the existence of $\sigma$ by backward induction. For $\tau$ the argument is similar and will be omitted for brevity. For $t=T-1$ we get $U_{T-1} \geq A_{\sigma}$ by putting $\sigma:=T$. Now suppose that for some $t=1, \ldots, T-1$ we have already established that there is a stopping time $\eta>t$ such that $U_{t} \geq A_{\eta}$. Let us put

$$
\sigma:=\eta \mathbf{1}_{\left\{A_{t}>U_{t}\right\}}+t \mathbf{1}_{\left\{A_{t} \leq U_{t}\right\}}
$$

It follows that

$$
U_{t-1} \geq U_{t} \wedge A_{t}=U_{t} \mathbf{1}_{\left\{A_{t}>U_{t}\right\}}+A_{t} \mathbf{1}_{\left\{A_{t} \leq U_{t}\right\}} \geq A_{\eta} \mathbf{1}_{\left\{A_{t}>U_{t}\right\}}+A_{t} \mathbf{1}_{\left\{A_{t} \leq U_{t}\right\}}=A_{\sigma},
$$

completing the proof of the claim.

Next we show that

$$
V_{t} \vee B_{t} \leq U_{t} \wedge A_{t}
$$

for each $t=0, \ldots, T$. Suppose that this were not so, and take the largest $t=0, \ldots, T$ such that (3) is violated. Since $U_{T}=A_{T}, V_{T}=B_{T}$ and $B_{T} \leq A_{T}$, it follows that $t<T$, and we have $V_{t+1} \vee B_{t+1} \leq U_{t+1} \wedge A_{t+1}$, which implies that $V_{t} \leq U_{t}$. Moreover, we know that $B_{t} \leq A_{t}$. Hence, for (3) to be violated, at least one of the following two inequalities would have to hold at some node $\lambda \in \Lambda_{t}$ :

(a) $V_{t}^{\lambda}>A_{t}^{\lambda}$. We know that there is a stopping time $\tau>t$ such that $B_{\tau} \geq V_{t}$, so $B_{\tau}>A_{t}$ on $\lambda$. In this case the strategy to buy a large enough position in stock for $A_{t}^{\lambda}$ at time $t$ and node $\lambda$, and to sell it for $B_{\tau}$ at time $\tau$ for any scenario belonging to $\lambda$ (and otherwise to do nothing) would be a combined-cost arbitrage opportunity. To be precise, such a strategy $(X, Y)$ could be defined as

$$
\left(X_{s}, Y_{s}\right):= \begin{cases}(0,0) & \text { for } \quad s=0, \ldots, t, \\ \mathbf{1}_{\lambda}\left(-A_{t} z-C_{t}, z\right) & \text { for } \quad s=t+1, \ldots, \tau, \\ \mathbf{1}_{\lambda}\left(-A_{t} z+B_{\tau} z-C_{t}-C_{\tau}, 0\right) & \text { for } \quad s=\tau+1, \ldots, T+1,\end{cases}
$$

for a large enough number $z>0$ such that $\left(-A_{t}+B_{\tau}\right) z>C_{t}+C_{\tau}$ on $\lambda$. This can be done because, on a finite probability space, the random variables $-A_{t}+B_{\tau}>0$ and $C_{t}+C_{\tau}$ take only finitely many values, hence there are constants $m, M>0$ such that $-A_{t}+B_{\tau}>m$ and $C_{t}+C_{\tau}<M$, and we can put $z:=M / m$. 
(b) $U_{t}^{\lambda}<B_{t}^{\lambda}$. We know that there is a stopping time $\sigma>t$ such that $A_{\sigma} \leq U_{t}$, so $A_{\sigma}<B_{t}$ on $\lambda$. The strategy $(X, Y)$ defined as

$$
\left(X_{s}, Y_{s}\right):= \begin{cases}(0,0) & \text { for } \quad s=0, \ldots, t \\ \mathbf{1}_{\lambda}\left(B_{t} z-C_{t},-z\right) & \text { for } \quad s=t+1, \ldots, \sigma \\ \mathbf{1}_{\lambda}\left(B_{t} z-A_{\sigma} z-C_{t}-C_{\sigma}, 0\right) & \text { for } \quad s=\sigma+1, \ldots, T+1\end{cases}
$$

would be a combined-cost arbitrage opportunity when $z>0$ is large enough so that $\left(B_{t}-A_{\sigma}\right) z>C_{t}+C_{\sigma}$ on $\lambda$. Such a number $z$ can be found in a similar manner as in (a).

This contradicts the assumption that there is no combined-cost arbitrage opportunity. Claim (3) has therefore been proved.

We are ready to construct a process $S$ and a family of single-step probability measures $Q$ by induction. At time $t=0$ we take any value

$$
S_{0} \in\left[V_{0} \vee B_{0}, U_{0} \wedge A_{0}\right]
$$

Now suppose that an $\mathscr{F}_{t}$-measurable random variable $S_{t} \in\left[V_{t} \vee B_{t}, U_{t} \wedge A_{t}\right]$ has already been constructed for some $t=0, \ldots, T-1$. For each $\lambda \in \Lambda_{t}$ we have

$$
V_{t+1}^{v} \vee B_{t+1}^{\nu}=V_{t}^{\lambda} \leq S_{t}^{\lambda} \leq U_{t}^{\lambda}=U_{t+1}^{\mu} \wedge A_{t+1}^{\mu}
$$

for some $\mu, v \in \operatorname{succ}(\lambda)$. If $\mu \neq v$, we put

$$
S_{t+1}^{\mu}:=U_{t+1}^{\mu} \wedge A_{t+1}^{\mu}, \quad S_{t+1}^{\nu}:=V_{t+1}^{v} \vee B_{t+1}^{v}
$$

and, for any $\eta \in \operatorname{succ}(\lambda)$ other than $\mu$ or $\nu$, we take as $S_{t+1}^{\eta}$ any value

$$
S_{t+1}^{\eta} \in\left[V_{t+1}^{\eta} \vee B_{t+1}^{\eta}, U_{t+1}^{\eta} \wedge A_{t+1}^{\eta}\right]
$$

This means that

$$
\min _{\mu \in \operatorname{succ}(\lambda)} S_{t+1}^{\mu} \leq S_{t}^{\lambda} \leq \max _{\mu \in \operatorname{succ}(\lambda)} S_{t+1}^{\mu}
$$

so there is a probability measure $Q_{t}^{\lambda}$ on the sigma-field $\lambda \cap \mathscr{F}_{t+1}$ such that (2) holds. But if $\mu=v$, then we put

$$
S_{t+1}^{\mu}:=S_{t}^{\lambda}
$$

and, for any $\eta \in \operatorname{succ}(\lambda)$ other than $\mu$, we take as $S_{t+1}^{\eta}$ any value

$$
S_{t+1}^{\eta} \in\left[V_{t+1}^{\eta} \vee B_{t+1}^{\eta}, U_{t+1}^{\eta} \wedge A_{t+1}^{\eta}\right]
$$

Moreover, we put $Q_{t}^{\lambda}(\mu):=1$ and $Q_{t}^{\lambda}(\eta):=0$ for any $\eta \in \operatorname{succ}(\lambda)$ other than $\mu$, which defines a probability measure $Q_{t}^{\lambda}$ on the sigma-field $\lambda \cap \mathscr{F}_{t+1}$ such that (2) 
holds. This construction produces an adapted process $S$ such that $B \leq S \leq A$, and a family of absolutely continuous single-step probability measures $Q$ such that $S$ is a martingale with respect to the family $Q$. The implication $(1) \Rightarrow(2)$ has been proved.

Conversely, to verify that (2) $\Rightarrow$ (1), we assume that condition (2) holds and, to argue by reductio ad absurdum, suppose that there is a combined-cost arbitrage opportunity $(X, Y)$. Condition (2) implies that

$$
X_{t}^{\lambda}+S_{t-1}^{\lambda} Y_{t}^{\lambda} \geq \min _{\mu \in \operatorname{succ}(\lambda)}\left(X_{t}^{\lambda}+S_{t}^{\mu} Y_{t}^{\lambda}\right)
$$

for each $t=1, \ldots, T$ and $\lambda \in \Lambda_{t-1}$. Next, since $(X, Y)$ is a combined-cost selffinancing strategy, it follows that, for each $t=0, \ldots, T$ and $\lambda \in \Lambda_{t}$,

$$
\begin{aligned}
X_{t}^{\lambda}- & X_{t+1}^{\lambda}+S_{t}^{\lambda}\left(Y_{t}^{\lambda}-Y_{t+1}^{\lambda}\right) \\
& \geq X_{t}^{\lambda}-X_{t+1}^{\lambda}+B_{t}^{\lambda}\left(Y_{t}^{\lambda}-Y_{t+1}^{\lambda}\right)^{+}-A_{t}^{\lambda}\left(Y_{t}^{\lambda}-Y_{t+1}^{\lambda}\right)^{-} \\
& \geq C_{t}^{\lambda}>0
\end{aligned}
$$

or

$$
X_{t}^{\lambda} \geq X_{t+1}^{\lambda}, \quad Y_{t}^{\lambda} \geq Y_{t+1}^{\lambda}
$$

Hence

$$
X_{t}+S_{t} Y_{t} \geq X_{t+1}+S_{t} Y_{t+1}
$$

for each $t=0, \ldots, T$. We can show by backward induction that

$$
X_{t+1}+S_{t} Y_{t+1} \geq 0
$$

for each $t=0, \ldots, T$. Clearly, (8) holds for $t=T$, given that $X_{T+1} \geq 0$ and $Y_{T+1} \geq 0$. Now suppose that (8) holds for some $t=1, \ldots, T$. Take any $\lambda \in \Lambda_{t-1}$. Then

$$
X_{t}^{\lambda}+S_{t-1}^{\lambda} Y_{t}^{\lambda} \geq \min _{\mu \in \operatorname{succ}(\lambda)}\left(X_{t}^{\lambda}+S_{t}^{\mu} Y_{t}^{\lambda}\right) \geq \min _{\mu \in \operatorname{succ}(\lambda)}\left(X_{t+1}^{\lambda}+S_{t}^{\mu} Y_{t+1}^{\lambda}\right) \geq 0
$$

where the first inequality holds by (4), the second by (7) and the last one by the induction hypothesis, completing the backward induction argument.

To proceed further, let us put

$$
t:=\max \left\{s=0, \ldots, T+1 \mid X_{0} \geq \cdots \geq X_{s} \text { and } Y_{0} \geq \cdots \geq Y_{s}\right\}
$$

Since $(X, Y)$ is a combined-cost arbitrage opportunity, we know that $X_{0}=Y_{0}=0$ and $X_{T+1}^{\lambda}>0$ for some $\lambda \in \Lambda_{T}$. If $t=T+1$, it would mean that $0=X_{0} \geq X_{T+1}^{\lambda}>0$, a contradiction. On the other hand, if $t \leq T$, then there would be a $\lambda \in \Lambda_{t}$ such that (6) fails, so (5) would have to hold, implying that

$$
X_{t}^{\lambda}+S_{t}^{\lambda} Y_{t}^{\lambda}>X_{t+1}^{\lambda}+S_{t}^{\lambda} Y_{t+1}^{\lambda} \geq 0
$$


where the last inequality follows from (8). However, that too is impossible as $X_{t} \leq$ $X_{0}=0$ and $Y_{t} \leq Y_{0}=0$. This contradiction completes the proof.

\section{Fixed costs}

A fixed-cost model involves two processes $S$ and $C$ adapted to the filtration $\mathscr{F}$, where $0<S<\infty$ represents the stock prices and $0<C<\infty$ the fixed transaction costs. This is a special case of the combined-cost model when the ask and bid prices coincide. Hence, fixed-cost solvent portfolios, fixed-cost self-financing strategies and fixed-cost arbitrage opportunities are covered by Definitions 1 and 2 with $A:=B:=S$. In this case Theorem 1 reduces to the following result.

Corollary 1 The following conditions are equivalent:

(1) There is no fixed-cost arbitrage opportunity in the model with stock prices $S$ and fixed costs $C$;

(2) There exists a family of absolutely continuous single-step probability measures $Q$ such that $S$ is a martingale with respect to $Q$.

This version of the Fundamental Theorem of Asset Pricing under fixed costs is similar to that obtained by Jouini et al. (2001). However, our method of proof (the proof of Theorem 1) is different. Moreover, the equivalent condition for the absence of fixed-cost arbitrage is expressed in terms of single-step measures only, whereas that in Jouini et al. (2001) relies on a larger family of measures.

As a consequence of Theorem 1, together with Corollary 1, we also obtain an alternative characterisation of the absence of combined-cost arbitrage in terms of an embedded arbitrage-free fixed-cost model. It resembles earlier results for proportional transaction costs, which involve embedding an arbitrage-free frictionless model; for example, see Roux (2011).

Corollary 2 The following conditions are equivalent:

(1) There is no combined-cost arbitrage opportunity in the model with ask and bid prices $A, B$ and fixed costs $C$;

(2) There exists a process $S$ adapted to the filtration $\mathscr{F}$ such that $B \leq S \leq A$ and the model with stock prices $S$ and fixed costs $C$ admits no fixed-cost arbitrage opportunity.

\section{Concluding remarks}

In this work the classical Fundamental Theorem of Asset Pricing due to Harrison and Pliska (1981) is extended to discrete market models with simultaneous fixed and proportional transaction costs and finite state space. This also extends later work on the Fundamental Theorem of Asset Pricing under proportional costs such as Jouini and Kallal (1995), Kabanov and Stricker (2001), Ortu (2001) and Roux (2011), and under fixed costs as in Jouini et al. (2001). 
Developments for models with infinite state space and/or continuous time and/or several assets are likely to follow. Moreover, as the Fundamental Theorem of Asset Pricing has now been established for markets with simultaneous fixed and proportional costs, it will inform research on pricing and hedging derivative securities in this setting.

Acknowledgements The authors would like to express their gratitude to the anonymous reviewer, whose constructive comments and suggestions helped to improve the clarity of the paper and to correct some mistakes.

Funding This work was partially funded by the Engineering and Physical Sciences Research Council (Grants EP/M508196/1, EP/M506680/1).

\section{Compliance with ethical standards}

Conflict of interest The authors declare that they have no conflict of interest.

Open Access This article is licensed under a Creative Commons Attribution 4.0 International License, which permits use, sharing, adaptation, distribution and reproduction in any medium or format, as long as you give appropriate credit to the original author(s) and the source, provide a link to the Creative Commons licence, and indicate if changes were made. The images or other third party material in this article are included in the article's Creative Commons licence, unless indicated otherwise in a credit line to the material. If material is not included in the article's Creative Commons licence and your intended use is not permitted by statutory regulation or exceeds the permitted use, you will need to obtain permission directly from the copyright holder. To view a copy of this licence, visit http://creativecommons.org/licenses/ by $/ 4.0 /$.

\section{References}

Bouchard, B.: No-arbitrage in discrete-time markets with proportional transaction costs and general information structure. Finance Stoch 10, 276-297 (2006)

Cherny, A.: General arbitrage pricing model: II-transaction costs. Séminaire de Probabilités XL. Lectures Notes in Mathematics, vol. 1899, pp. 447-461. Berlin: Springer (2007)

Dalang, R.C., Morton, A., Willinger, W.: Equivalent martingale measures and no-arbitrage in stochastic securities market models. Stoch Stoch Rep 29, 185-201 (1990)

Delbaen, F., Schachermayer, W.: The fundamental theorem of asset pricing for unbounded stochastic processes. Math Ann 312, 215-250 (1994)

Delbaen, F., Schachermayer, W.: The Mathematics of Arbitrage. Berlin: Springer (2006)

Denis, E., Kabanov, Y.: Consistent price systems and arbitrage opportunities of the second kind in models with transaction costs. Finance Stoch 16, 135-154 (2012)

Dolinsky, Y., Soner, Y.M.: Robust hedging with proportional transaction costs. Finance Stoch 18, 327-347 (2014)

Guasoni, P.: No arbitrage under transaction costs, with fractional Brownian motion and beyond. Math Finance 16, 569-582 (2006)

Guasoni, P., Rásonyi, M., Schachermayer, W.: The fundamental theorem of asset pricing for continuous processes under small transaction costs. Ann Finance 6, 157-191 (2010)

Guasoni, P., Lépinette, E., Rásonyi, M.: The fundamental theorem of asset pricing under transaction costs. Finance Stoch 16, 741-777 (2012)

Grigoriev, P.: On low dimensional case in the fundamental asset pricing theorem under transaction costs. Stat Decis 23, 33-48 (2005)

Harrison, J.M., Pliska, S.R.: Martingales and stochastic integrals in the theory of continuous trading. Stoch Process Appl 11, 215-260 (1981)

Jouini, E., Kallal, H.: Martingales and arbitrage in securities markets with transaction costs. J Econ Theory 66, 178-197 (1995)

Jouini, E., Kallal, H., Napp, C.: Arbitrage and viability in securities markets with fixed trading costs. J Math Econ 35, 197-221 (2001) 
Kabanov, Y.M.: Hedging and liquidation under transaction costs in currency markets. Finance Stoch $\mathbf{3}$, 237-248 (1999)

Kabanov, Y., Safarian, M.: Markets with Transaction Costs: Mathematical Theory. Berlin: Springer (2009)

Kabanov, Y.M., Stricker, C.: The Harrison-Pliska arbitrage pricing theorem under transaction costs. J Math Econ 35, 185-196 (2001)

Kabanov, Y.M., Rásonyi, M., Stricker, C.: No-arbitrage criteria for financial markets with efficient friction. Finance Stoch 6, 371-382 (2002)

Lepinette, E., Tran, T.: General financial market model defined by a liquidation value. Stochastics $\mathbf{8 8}$, 437-459 (2016)

Lepinette, E., Tran, T.: Arbitrage theory for non convex financial market models. Stoch Process Appl 127, 3331-3353 (2017)

Ortu, F.: Arbitrage, linear programming and martingales in securities markets with bid-ask spreads. Decis Econ Finance 24, 79-105 (2001)

Rola, P.: Arbitrage in markets with bid-ask spreads. The fundamental theorem of asset pricing in finite discrete time markets with bid-ask spreads and a money account. Ann Finance 11, 453-475 (2015)

Roux, A.: The fundamental theorem of asset pricing in the presence of bid-ask and interest rate spreads. $\mathrm{J}$ Math Econ 47, 159-163 (2011)

Schachermayer, W.: The fundamental theorem of asset pricing under proportional transaction costs in finite discrete time. Math Finance 14, 19-48 (2004)

Zhang, S., Xu, C., Deng, X.: Dynamic arbitrage-free asset pricing with proportional transaction costs. Math Finance 12, 89-97 (2002)

Zhao, J., Lepinette, E.: The Dalang-Morton-Willinger version of the fundamental theorem of asset pricing for the bid-ask financial market model (2018). https://hal.archives-ouvertes.fr/hal-01666860v4. Accessed 23 March 2018

Publisher's Note Springer Nature remains neutral with regard to jurisdictional claims in published maps and institutional affiliations. 